\title{
Leidenfrost mixer
}

Hideyuki Sugioka, ${ }^{1, a)}$ Mako Kubota, ${ }^{1}$ and Satoru Segawa ${ }^{1}$

${ }^{1}$ Department of Mechanical Systems Engineering, Shinshu University 4-17-1

Wakasato, Nagano 380-8553, Japan

(Dated: 2 March 2019)

Mixing is essential for various microfluidic applications. In this study, we propose a mixer using Leidenfrost phenomena. That is, by using a pair of ratchets (whose directions are opposite each other), we showed that the Leidenfrost mixer can rotate a water droplet with the angular velocity of $\sim 30 \mathrm{rad} / \mathrm{s}$ and can mix the pearl pigment (whose diffusion coefficient is zero) rapidly.

PACS numbers: 47.57.-s, 85.90.+h, 82.45.Hk, 83.50.Lh

a) Electronic mail: hsugioka@shinshu-u.ac.jp 
Mixing is essential for various microfluidic applications, such as a total analysis system $(\mu \mathrm{TAS})$ and a Lab-on-a-chip (LOC), and a rotation of water plays an important role since it enhances a mixing phenomenon even in a low Reynolds number (low-Re) regime, although the mixing phenomenon is achieved only through diffusion ${ }^{1,2}$. Specifically, Stroock et al. ${ }^{1}$ showed that a required mixing length of the mixer using a spiral rotation is proportional to not the Péclet number (Pe) but logarithm of Pe in a low-Re regime. However, to generate a high-speed rotation of water is often difficult in microfluidic systems since a mechanical pump results in increasing of the size of the systems. Further, the flow velocity of a nonmechanical pump, such as an electro-osmotic pump, is very slow and it is typically $\sim 1 \mathrm{~mm} / \mathrm{s}$ at most $^{3}$. Thus, a high-speed simple mixer is required for innovative microfluidic systems.

In 2006, Linke et al. ${ }^{4}$ reported that the hot surface having a ratchet-like topology can propel a levitating Leidenfrost droplet with high velocities of the level of $\sim 5 \mathrm{~cm} / \mathrm{s}^{4}$ and the self-propelled device using a Leidenfrost phenomenon, which is a kind of film boiling 5,6 , has received special attention in this decade ${ }^{6}$. In particular, Wells et al. showed that a levitating dry-ice block rotates on a hot turbine-like surface owing to the Leidenfrost effect ${ }^{7}$. Mrinal et al. showed that a Leidenfrost droplet on a ratchet has a combined motion of translation and self-rotation ${ }^{8}$. Bouillant et al. reported that Leidenfrost droplets on a horizon substrates self-rotate and self-propel in the direction they are rolling as a wheel ${ }^{9}$. However, a mixer using Leidenfrost phenomena has not been reported yet, although Wells et al. argued that a water film layer under the circular metal disc also rotates on a hot turbine-like surface owing to the Leidenfrost effect from the view point of energy harvesting ${ }^{7}$.

Thus, in this study, we focus on the mixer using Leidenfrost phenomena and experimentally prove the performance. In particular, we here report that the Leidenfrost mixer using a pair of ratchets can rotate a water droplet with a angular velocity of $\sim 30 \mathrm{rad} / \mathrm{s}$ and it can mix the pigment (whose diffusion coefficient $D$ is zero) rapidly.

Figure 1 shows a experimental setup of the Leidenfrost mixer. As shown in Fig. 1(a), we put a pair of hot aluminum plates (having a ratchet-like topology) on a hot plate [whose surface temperature is $\left.T_{s, 2}\left(\geq 240^{\circ} \mathrm{C}\right)\right]$ in the opposite direction to each other and put a hot brass ring (of diameter $L=10 \mathrm{~mm}$ and height $h^{\prime}=0.9 \mathrm{~mm}$ ) on the middle of the ratchets (whose surface temperature is $T_{s, 1}$ ). Here, the direction of the ratchet is defined as the inclined direction of the ratchet tooth and the ring guides water. Since the droplet has a tendency to move in the inclined direction, the left side water of the droplet moves in 
the downward direction, whereas the right side water moves in the upward direction. As a result, the levitating Leidenfrost droplet can rotate in the counter-clockwise direction on the ratchets, as shown in Fig. 1(a). Figure 1(b) shows the side view of the water rotating device. As shown in Fig. 1(b), we poured water into the pre-heated ratchet surface enclosed by the ring until the height $h$ of the droplet becomes the initial height $h_{i}(\sim 4 \mathrm{~mm})$ and observed the rotatory motion of the water droplet. In Fig. 1(b), we consider that a Leidenfrost vapor layer exists not only on the ratchet but also on the side of the hot ring and thus the existence of the hot ring plays an important role to obtain a high-speed rotation of the water droplet. Further, by using video data of size $1280 \times 720$ with a frame rate of 240 or 120 fps, we determined the angular velocity $\omega(t)$ at time $t$.

Figure 2 shows the photographs of the mixing phenomenon of the pearl pigment of $D=0$ $\mathrm{m}^{2} / \mathrm{s}$ at $T_{s, 2}=300{ }^{\circ} \mathrm{C}$. Here, Péclet number Pe $(\sim U L / D)$ of the system is infinite and Re $(\sim U L / \nu)$ is approximately 1200 , where $U(\sim 0.12 \mathrm{~m} / \mathrm{s})$ is the representative velocity and $\nu\left(10^{-6} \mathrm{~m}^{2} / \mathrm{s}\right.$ for water $)$ is the kinematic viscosity. As shown in Figs. $2(\mathrm{a})$ to $2(\mathrm{~d})$, the pigment was mixed rapidly (within $0.53 \mathrm{~s}$ ) by the Leidenfrost mixer, whereas we could not observe any diffusion of the pigment in water in a stationary state. Specifically, the water droplet was transparent at $t=0 \mathrm{~s}$, as shown in Fig. 2(a) and then the white powder of the pigment poured on a surface of the water at the bottom position transported along to the water flow in an early stage. Soon we observed that the pigment spread to the crescentshaped region at $t=0.19 \mathrm{~s}$, as shown in Fig. 2(b). Further, the pigment spread to the doughnut-shaped region at $t=0.33 \mathrm{~s}$ in Fig. 2(c) and then the whole area becomes white at $t=0.53 \mathrm{~s}$ in Fig. 2(d).

Figures. 2(e) to 2(h) show the apparent flow fields (or trajectories) of the pearl pigment obtained by the particle image velocimetry (PIV) using the image correlation method. As shown in Figs. 2(f) and 2(g), we could find rotational but complex flows in a transient stage, although we could not visualize the flow of the particles or water under the complete mixing state or the complete non-mixing state as shown in Figs. 2(e) and 2(h). Further, to evaluate the mixing performance quantitatively, we defined the stirring index $\eta_{\text {mix }}$ as $\eta_{\text {mix }} \equiv \frac{I_{t=t}-I_{t=0}}{I_{t=\infty}-I_{t=0}}$, where $I$ is the intensity of the pixel of the image. Figure 2(i) shows the dependence of $\eta_{\text {mix }}$ on $t$ at the center position. As shown in Fig. 2(i), $\eta_{\text {mix }}$ at the center increases rapidly at $t \simeq 0.2 \mathrm{~s}$ and becomes $\sim 1$ at $\simeq 0.6 \mathrm{~s}$ as expected. Further, Fig. 2(j) shows an apparent flow field for the side view image of our mixer. Although the apparent 
flow field just shows the surface flow from the left to the right, we consider that the inner bottom particle in the front region has a tendency to move in the upper right direction owing to the effect of the straight ratchet and the obstacle of the ring wall, as illustrated in the upper part in Fig. 2(j). Note that we observed a vertical rotation for a water droplet on a single ratchet as shown in Fig. 2(k). Thus, we consider that the water in the front region has a tendency to rotate vertically in the counter-clockwise direction, whereas the water in the rear region has a tendency to rotate vertically in the clockwise direction. As a result, our mixer can mix the particles effectively when particles move across the border between two ratchets, although it has not visualize well in this experiment.

Figure 3 shows the angular velocity $\omega$ of the Leidenfrost mixer. Here, to measure the values of $\omega$, we put a square paper of size $L^{\prime} \times L^{\prime}$, whose mass per unit area $\rho^{\prime}$ is $1.0 \times 10^{-4}$ $\mathrm{g} / \mathrm{mm}^{2}$ and whose mass $m^{\prime}$ is $\rho^{\prime} L^{\prime 2}$ (i.e., $m^{\prime}=0.0025 \mathrm{~g}$ at $L^{\prime}=5 \mathrm{~mm}$ ). Figure 3(a) shows the dependences of $\omega$ on $t$ at $T_{s, 2}=300{ }^{\circ} \mathrm{C}$. Here, we performed the same experiments $n_{t}$ $(=3)$ times under the same condition and $n$ is the number of experiments. In Fig. 3(a), the maximum angular velocity $\omega_{\max }$ is approximately $30 \mathrm{rad} / \mathrm{s}$ and the rotation time $t_{\text {rot }}$ is approximately $30 \mathrm{~s}$. Here, the rotation of the paper stopped before the complete evaporation by the contact between the paper and the ring. Although there is a large variation in the data of $\omega_{\max }$ and $t_{\text {rot }}$, we consider that the variation will be controlled in the future by controlling a variation of $h_{i}$ more precisely. Further, since $\omega_{\max } \sim 30 \mathrm{rad} / \mathrm{s}$, the maximum flow velocity $\left(\sim \omega_{\max } L^{\prime}\right)$ is approximately $0.15 \mathrm{~m} / \mathrm{s}$. Note that the maximum apparent velocity by the PIV method $\left(u_{\max }^{\text {piv }}\right)$ is $5.6 \mathrm{~cm} / \mathrm{s}$ [in Fig. $\left.2(\mathrm{f})\right]$ at $r \simeq \frac{L}{4}=2.5 \mathrm{~mm}$, where $r$ is the distance from the center of the mixer. Thus, the angular velocity of the fluid by the PIV $\left(\omega_{\text {piv }} \simeq u_{\max }^{p i v} /\left(\frac{L}{4}\right)\right)$ is approximately $22.4 \mathrm{rad} / \mathrm{s}$. Since there is a tendency that $u_{\text {max }}^{p i v}$ becomes smaller than the real flow velocity of the fluid, we can consider that the angular velocity determined from the motion of a paper approximately agrees with that of the fluid rotation $(\sim 30 \mathrm{rad} / \mathrm{s})$, even though the mixing in a droplet requires an inhomogeneous rotation of fluid due to a turbulence flow component.

Figure 3(b) shows the dependence of $\omega$ on $t$ at $T_{s, 2}=240$ to $300{ }^{\circ} \mathrm{C}$. The rotation of water was observed at $T_{s, 2} \geq 240{ }^{\circ} \mathrm{C}\left(T_{s, 1} \geq 225{ }^{\circ} \mathrm{C}\right)$ and the temperature difference $\Delta T_{s}=T_{s, 2}-T_{s, 1}=5$ to $20 \mathrm{~K}$ in the range that $200 \leq T_{s, 2} \leq 300{ }^{\circ} \mathrm{C}$. In Fig. $3(\mathrm{~b})$, the behavior of $\omega(t)$ at $T_{s, 2}=240{ }^{\circ} \mathrm{C}$ is unstable since it is in a nucleate boiling state, although $\omega_{\max }$ at $T_{s, 2}=240{ }^{\circ} \mathrm{C}$ is larger than that at the other temperature. Note that the plot 
for $T_{s, 2}=300{ }^{\circ} \mathrm{C}$ in Fig. 3(b) does not agree with any plots shown in Fig. 3(a), since we conducted anther experimental run of $n_{t}=3$ in addition to three experimental runs shown in Fig. 3(a). Figure 3(c) shows the dependence of $\omega_{\max }$ on $T_{s, 2}$. From Fig. 3(c), we find that $\omega_{\text {max }}$ has the maximum around $T_{s, 2}=280{ }^{\circ} \mathrm{C}$ in the range $250 \leq T_{s, 2} \leq 300{ }^{\circ} \mathrm{C}$. Since angular velocity induced by the Leidenfrost effect fluctuates significantly depending on experimental runs as shown in Fig. 3(a), the experiment of $n_{t}=3$ is not enough to conclude something. However, since Ok et al. ${ }^{10}$ already showed that there exists an optimum temperature for the mean velocity of the droplet on the ratchet (by the detail experiments), we consider that there exists an optimum temperature around $280{ }^{\circ} \mathrm{C}$ for the rotation in our system.

Although Wells et al. reported that a metal disc having a water film rotates on a hot turbine-like surface ${ }^{7}$, we first propose a high-speed mixer using Leidenfrost phenomena and showed the eminent mixing performance; i.e., our proposed mixer can mix the pigment of $D=0$ rapidly. In particular, the Leidenfrost mixer has remarkable merits. (1) In spite of its simple structure, it has a large flow velocity of the level of $0.1 \mathrm{~m} / \mathrm{s}$, which is approximately 100 times of the velocity of the mixer using electrokinetic phenomena (typically, $\sim 1 \mathrm{~mm} / \mathrm{s}$ ). Thus we can increase Re to approximately 100 times; e.g., we obtain $R e=1000$ and 10 for $L=10$ and $0.1 \mathrm{~mm}$, respectively and thus we can expect the mixing phenomena due to a turbulent flow (at least for $R e=1000$ ), where we assume that the kinematic viscosity $\nu=10^{-6} \mathrm{~m}^{2} / \mathrm{s}$ and the representative velocity $U=0.1 \mathrm{~m} / \mathrm{s}$. (2) By using the two-way straight ratchet structure with a ring, we can expect complex trajectories of particles which may accelerate a mixing phenomenon even in a low-Re regime. In particular, we consider that owing to the rapid change of the flow direction, the particles can spread rapidly compare to turbine-like surface. However, there still remains problems to overcome for the real microfluidic applications. For example, (i) rapid heating systems having the heat flux of more than $1 \mathrm{GW} / \mathrm{m}^{2}$ are probably required to generate a Leidenfrost phenomenon and to control our device at will. (ii) More efficient structure or driving method (e.g., a chaotic mixer that uses two vortex flows ${ }^{11}$ ) should be explored in this Leidenfrost mixer since $R e=10$ at $L=0.1 \mathrm{~mm} / \mathrm{s}$ sometimes may not be enough to obtain a complete mixing; in addition, we need to clarify the mixing performance in a low-Re regime. (iii) Closed chip system along with a vapor removing device for the Leidenfrost mixer should be investigated to obtain an safe microfluidic chip system.

In conclusion, we have shown that the Leidenfrost mixer can rotate water with high 
angular velocities of $\sim 30 \mathrm{rad} / \mathrm{s}$ and can mix the pigment whose diffusion coefficient is zero rapidly (within $0.6 \mathrm{~s}$ ).

This work was partially supported by JSPS KAKENHI Grant Number JP16K05650.

\section{REFERENCES}

${ }^{1}$ A. D. Stroock, S. K. W. Dertinger, A. Ajdari, I. Mezic, H. A. Stone, and G. M. Whitesides, Science 295, 647 (2002).

${ }^{2}$ C.-Y. Lee, C.-L. Chang, Y.-N. Wang, and L.-M. Fu, International Journal of Molecular Sciences 12, 3263 (2011).

${ }^{3}$ J. P. Urbanski, T. Thorsen, J. A. Levian, and M. Z. Bazant, Appl. Phys. Lett. 89, 143508 (2006).

${ }^{4}$ H. Linke, B. J. Alemán, L. D. Melling, M. J. Taormina, M. J. Francis, C. C. Dow-Hygelund, V. Narayanan, R. P. Taylor, and A. Stout, Phys. Rev. Lett. 96, 154502 (2006).

${ }^{5}$ A.-L. Biance, C. Clanet, and D. Qur, Physics of Fluids 15, 1632 (2003).

${ }^{6}$ D. Quéré, Annual Review of Fluid Mechanics 45, 197 (2013).

${ }^{7}$ G. G. Wells, R. Ledesma-Aguilar, G. McHale, and K. Sefiane, Nature Communications 6, 639 (2015).

${ }^{8}$ M. Mrinal, X. Wang, and C. Luo, Langmuir 33, 6307 (2017).

${ }^{9}$ A. Bouillant, T. Mouterde, P. Bourrianne, A. Lagarde, C. Clanet, and D. Quéré, Nature Physics 10, 1038 (2018).

${ }^{10}$ J. T. Ok, E. Lopez-Oña, D. E. Nikitopoulos, H. Wong, and S. Park, Microfluidics and Nanofluidics , 1045 (2011).

${ }^{11}$ H.Aref, J. Fluid Mech. 143, 1 (1984). 


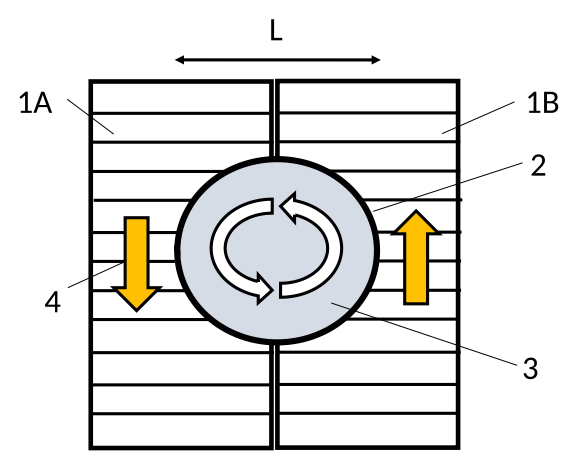

(a) Top view

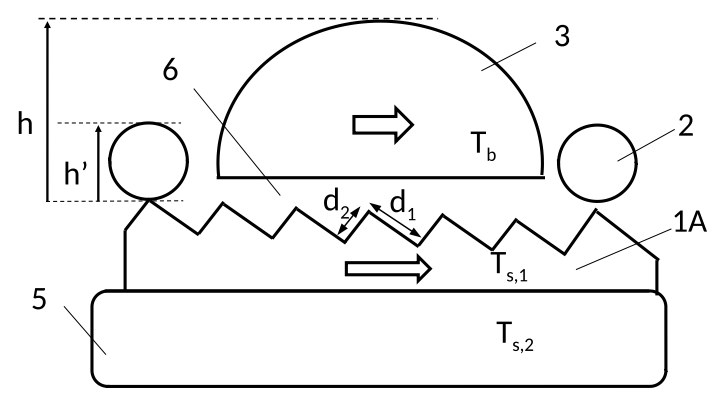

(b) Side view

FIG. 1. (Color online) Experimental setup of the rotation of water using Leidenfrost phenomena.

1A, 1B: hot ratchet, 2: hot ring, 3: water droplet, 4: ratchet direction, 5: hot plate, and 6: Leidenfrost vapor layer. Here, $T_{b}=100{ }^{\circ} \mathrm{C}, T_{s, 2}=240$ to $300{ }^{\circ} \mathrm{C}, d_{1}=1.2 \mathrm{~mm}, d_{2}=0.3 \mathrm{~mm}$, $L=10 \mathrm{~mm}, h^{\prime}=0.9 \mathrm{~mm}$, and $h_{i} \simeq 4 \mathrm{~mm}$. 


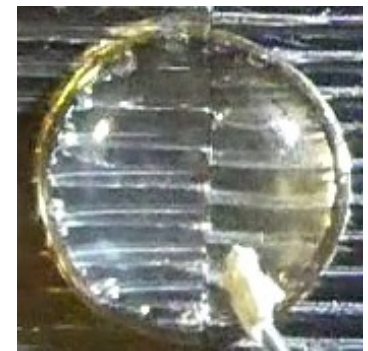

(a) $t=0 \mathrm{~s}$

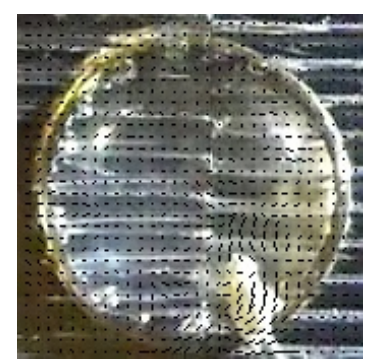

(e) $t=0 \mathrm{~s}$

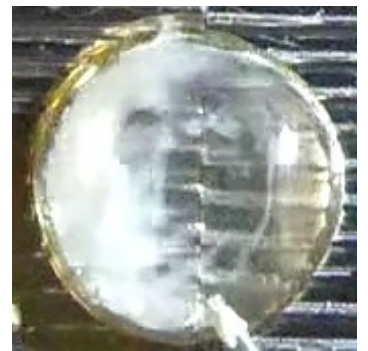

(b) $t=0.19 \mathrm{~s}$

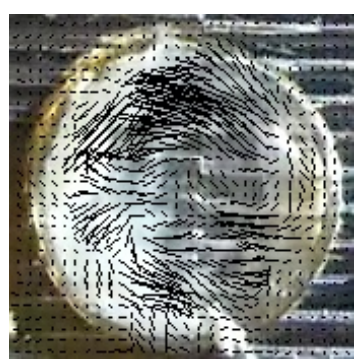

(f) $t=0.19 \mathrm{~s}$

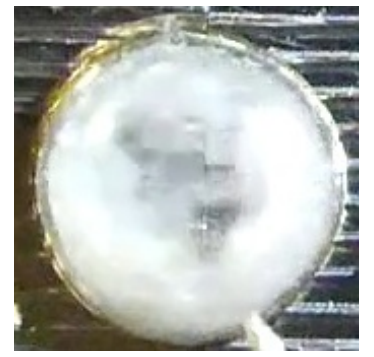

(c) $t=0.33 \mathrm{~s}$

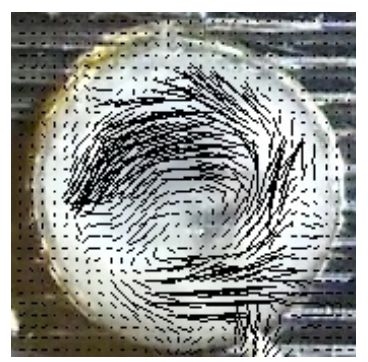

(g) $t=0.33 \mathrm{~s}$

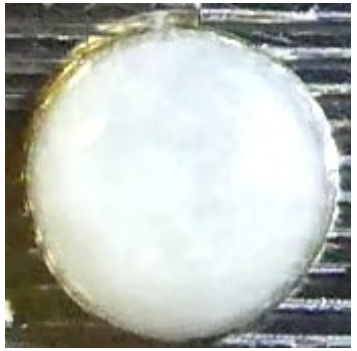

(d) $t=0.53 \mathrm{~s}$

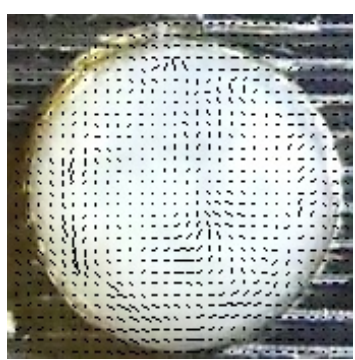

(h) $t=0.53 \mathrm{~s}$

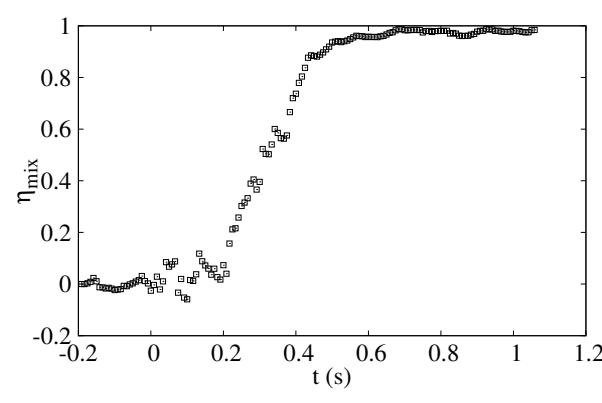

(i) Dependence of $\eta_{m i x}$ on $t$

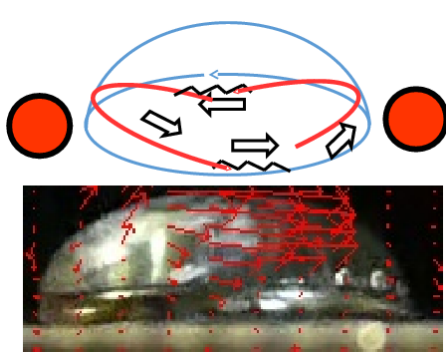

(j) Side view of the double

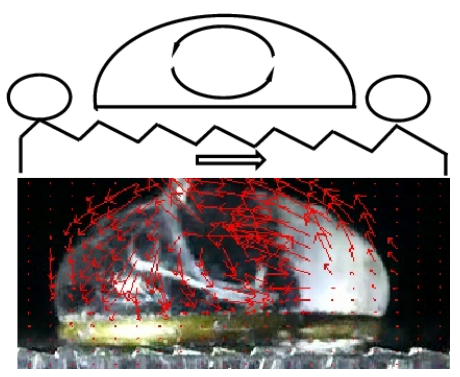

(k) Vertical rotation on the ratchet mixer $(t=0.17 \mathrm{~s})$ and $\quad$ single ratchet $(t=0.21 \mathrm{~s})$

the flow model

FIG. 2. (Color online) Rapid mixing phenomenon of the pearl pigment whose diffusion coefficient is zero. Her, $D=0, P e=\infty, R e \sim 1200, U \sim 0.12 \mathrm{~m} / \mathrm{s}, L=10 \mathrm{~mm}, h_{i} \simeq 4 \mathrm{~mm}$, and $T_{s, 2}=300$ ${ }^{\circ} \mathrm{C}$. As shown in figures, the pigment of $D=0$ is mixed immediately (within $0.6 \mathrm{~s}$ ). The maximum apparent velocities using PIV $\left(u_{\text {max }}^{\text {piv }}\right)$ at $t=0.0,0.19,0.33$, and 0.53 are $0.0,5.6,4.5$, and $1.7 \mathrm{~cm} / \mathrm{s}$, respectively. 


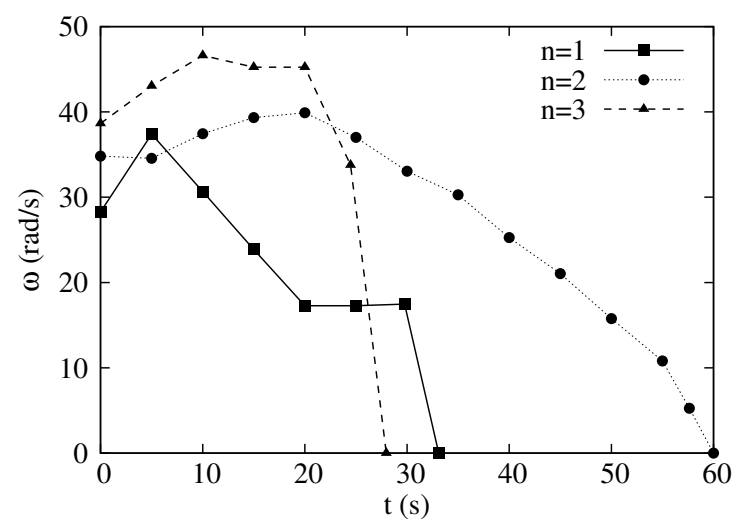

(a) Dependence of $\omega$ on $t$ at $T_{s, 2}=300{ }^{\circ} \mathrm{C}$

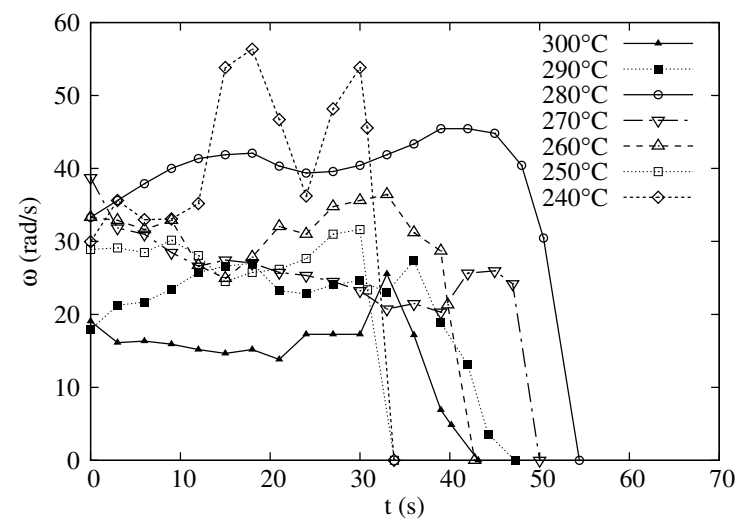

(b) Dependence of $\omega$ on $t$ at $T_{s, 2}=240$ to $300^{\circ} \mathrm{C}$

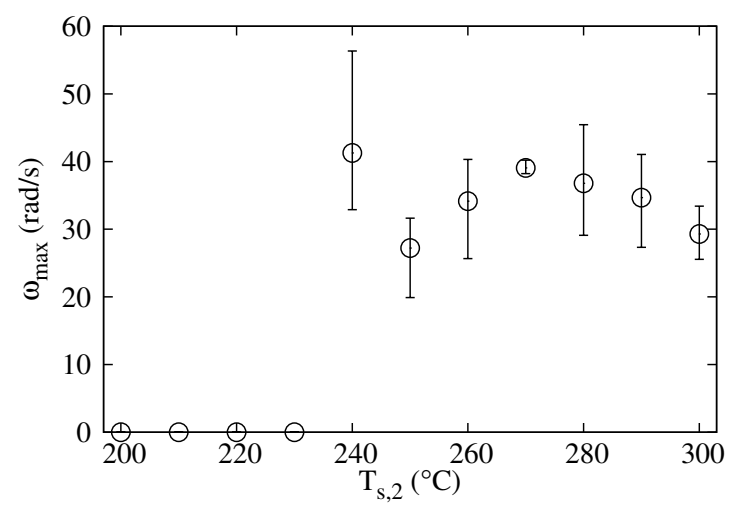

(c) Dependence of $\omega_{\max }$ on $t\left(n_{t}=3\right)$

FIG. 3. Angular velocity of the Leidenfrost mixer. Here, $L=10 \mathrm{~mm}, L^{\prime}=5 \mathrm{~mm}$, and $h_{i} \simeq 4$ $\mathrm{mm}$. 